\title{
Stellar Archaeology: Using Metal-Poor Stars to Test Theories of the Early Universe
}

\author{
Anna Frebel, Jarrett L. Johnson and Volker Bromm \\ McDonald Observatory and Department of Astronomy, University of Texas, \\ 1 University Station, C1402, Austin TX, 78712 \\ email: anna, jljohnson, vbromm@astro.as.utexas.edu
}

\begin{abstract}
Constraints on the chemical yields of the first stars and supernova can be derived by examining the abundance patterns of different types of metal-poor stars. We show how metal-poor stars are employed to derive constraints of the formation of the first low-mass stars by testing a fine-structure line cooling theory. The concept of stellar archaeology, that stellar abundances truly reflect the chemical composition of the earliest times, is then addressed. The accretion history of a sample of metal-poor stars is examined in detail in a cosmological context, and found to have no impact on the observed abundances. Predictions are made for the lowest possible $\mathrm{Fe}$ and $\mathrm{Mg}$ abundances observable in the Galaxy, $[\mathrm{Fe} / \mathrm{H}]_{m i n}=-7.5$ and $[\mathrm{Mg} / \mathrm{H}]_{\text {min }}=-5.5$. The absence of stars below these values is so far consistent with a topheavy IMF. These predictions are directly relevant for future surveys and the next generation of telescopes.
\end{abstract}

Keywords. stars: abundances, stars: Population II, Galaxy: halo, early Universe

\section{Introduction}

The first stars, the so-called Population III (Pop III), were the key drivers of early cosmic evolution. They produced large amounts of hydrogen-ionizing radiation which initiated the reionization of the universe. The first supernova (SN) explosions then provide the pristine intergalactic medium (IGM) with the first heavy elements. Based on numerical simulations, the current theoretical models of Pop III star formation suggests that the Pop III initial mass function (IMF) was top-heavy (Bromm, Coppi \& Larson 2002). Testing this crucial prediction is one of the main goals of the upcoming James Webb Space Telescope (JWST), but it is important to apply complementary probes that are accessible already now.

We here consider a "near-field cosmology" by assessing low-mass, metal-poor Galactic halo stars as probes of the nucleosynthetic signature of the first stars. The most metalpoor Galactic halo stars are now frequently used in an attempt to reconstruct the onset of the chemical and dynamical formation processes of the Galaxy. These stars are an easily-accessible local equivalent of the high-redshift Universe, and are used to address near-field cosmological questions.

\section{Testing a Low-Mass Star Formation Theory}

Large samples of these old, metal-poor objects are now being employed to test theoretical predictions about the early Universe. Compared to the first stars that presumably were very massive $\left(100 \mathrm{M}_{\odot}\right)$, the most metal-poor stars are of low mass $\left(M<1 M_{\odot}\right)$. Currently, two competing scenarios (fine-structure line cooling and dust cooling) responsible for cooling of the early interstellar medium are being debated in the literature. 
Fine-structure line cooling through C I and O II may be responsible for sufficient fragmentation of near-primordial gas which led to the formation of the first low-mass stars (Bromm \& Loeb 2003). We have developed an "observer-friendly" formulation of the fine-structure line cooling theory (Frebel, Johnson \& Bromm (2007)) that incorporates the observed $\mathrm{C}$ and/or $\mathrm{O}$ abundances of metal-poor stars; $D_{\text {trans }}=\log \left(10^{[\mathrm{C} / \mathrm{H}]}+0.3 \times\right.$ $\left.10^{[\mathrm{O} / \mathrm{H}]}\right) \geqslant-3.5$. If this theory is correct, a low-mass star that is still observable today, thus has to lay above the critical metallicity limit of $D_{\text {trans }}=-3.5$. The observations thus far support this theory, and furthermore we predict that future stars to be found with $[\mathrm{Fe} / \mathrm{H}]<-4.0$ will show significant $\mathrm{C}$ and/or $\mathrm{O}$ overabundances.

We have further populated the Figure 1 of Frebel, Johnson \& Bromm (2007) with lower limits for $D_{\text {trans }}$, based on new $[\mathrm{Fe} / \mathrm{H}]$ and $[\mathrm{C} / \mathrm{Fe}]$ data of various stars from the literature. Among them is $\mathrm{HE} 0558-4840$ with $[\mathrm{Fe} / \mathrm{H}]=-4.8$. Its $\mathrm{C}$ abundance provides a lower limit which lies somewhat below the $D_{\text {trans }}$ limit. The $\mathrm{O}$ abundance, which is not yet available, will determine if the star will remain below the limit. Within the theoretical uncertainties, however, HE 0557-4840 still does not violate the fine-structure line cooling theory. More such "borderline" examples are crucial for a successful test of this theory. We also note that this is one of the few theoretical works that actually can be confirmed or refuted with observational data. We therefore encourage observers to add their $\mathrm{C}$ and/or O measurements of metal-poor stars to such a plot in order to fully map the observable regions, and to test the fine-structure line theory of Bromm \& Loeb (2003) with the most solid observational material. We are currently studying new carbon-poor stars from the bright metal-poor sample of Hamburg/ESO survey which will then be added to our diagram.

\section{Validating Stellar Archaeology}

Abundances of metal-poor stars are primarily used to infer details of the chemical composition of the early Universe and the onset of Galactic chemical evolution. It is hereby assumed that the observed abundances reflect the conditions of the interstellar medium at the time and place of their formation. Accretion of material from the interstellar medium while a star orbits in the Galaxy for $~ 10$ Gyr has long been suggested to affect the observed abundance patterns, although only simplified calculations have been carried out. Iben (1983) calculated a basic "pollution limit" of $[\mathrm{Fe} / \mathrm{H}]=-5.7$. Based on Bondi-Hoyle accretion, he predicted that no stars could be found with Fe abundances below this value because they would have accreted too much material from the ISM.

To assess the potential accretion history of individual halo stars in more detail, we carried out a kinematic analysis of metal-poor stars with $[\mathrm{Fe} / \mathrm{H}]<-2.5$ selected from the Sloan Digital Sky Survey (DR6). The necessary input data (e.g., abundances, radial velocities, proper motions, distances) are available on the DR6 website†. All stars were followed for $10 \mathrm{Gyr}$ in a three component potential adopted from Johnston (1998). Accretion was assumed to take place only during disk crossings, whereby the disk height was set to $100 \mathrm{pc}$ with a density of $n=5 \mathrm{~cm}^{-3}$. The amount of accreted $\mathrm{Fe}$ and $\mathrm{Mg}$ are calculated based on the total accreted amount for every crossing. A simple model for chemical evolution is taken into account in which the interstellar medium has scaled solar abundances. In Figure 1, we compare the total accreted Mg amount for every star with the observed abundances. The stars all have lower "accreted abundances" than observed abundances, sometimes by several dex. This generally confirms that accretion does not

$\dagger$ http://www.sdss.org/dr6/ 


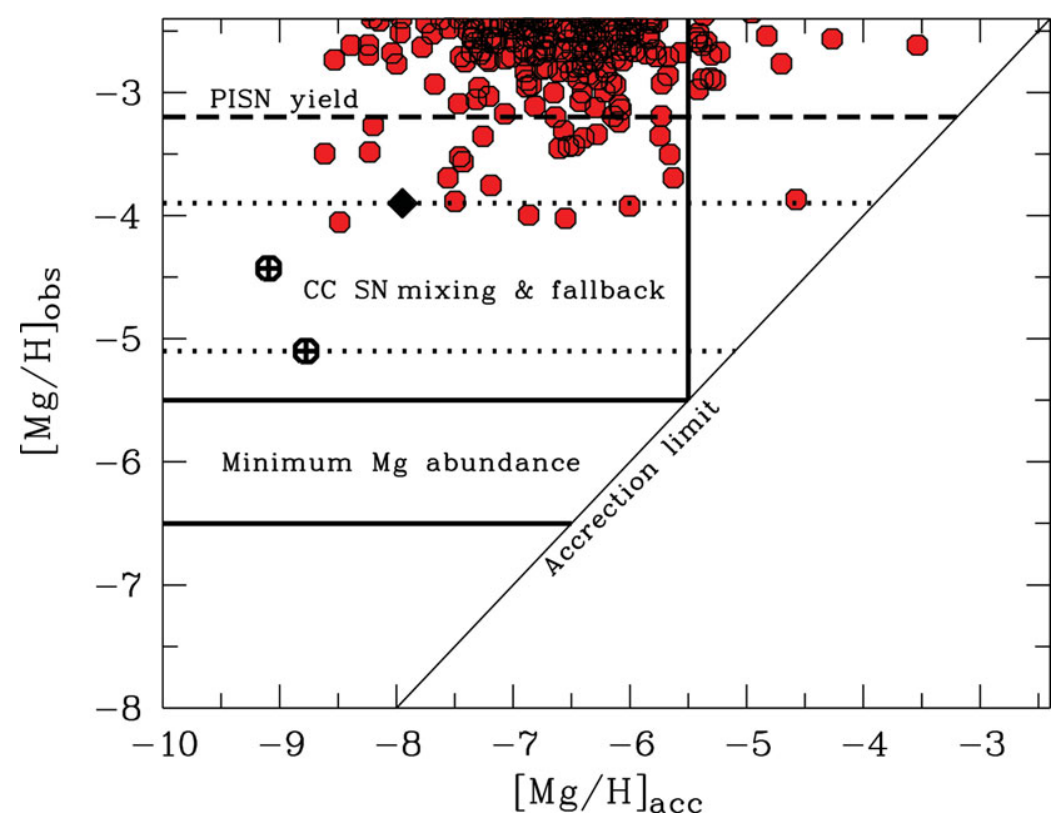

Figure 1. Observed vs. "accreted" $\mathrm{Mg}$ abundances for the sample of metal-poor stars (red circles). The three most Fe-poor stars are indicated (crossed circles, diamond). All stars have accreted less metals ( $>$ one order of magnitude) than what is observed, thus validating stellar archaeology. The minimum $\mathrm{Mg}$ abundance range, calculated under the assumption of a top-heavy Pop III IMF, is indicated. The Mg abundances arising from a PISN event in an atomic cooling halo is also shown (dashed line), Mg levels of pre-enrichment from a $25 M_{\odot}$ mixing and fallback SN (dotted lines). The stars left of the vertical line that can be used to place constraints on the Pop III IMF, where accretion cannot affect whether they lie above or below the minimum abundance range, predicted for a top-heavy IMF.

dominate the abundance pattern of old metal-poor stars. The same is found in the case of Fe.

We also investigated the special case where each star is moving once through a dense molecular cloud. In this case, the observed abundance pattern might be dominated by the signature of the interstellar medium. We find, however, that the stars still have slightly lower "accreted abundances" than observed abundances. Whether every star indeed runs through such a cloud remains unclear, but even if it does, the accretion process heavily depends on the space velocity of a star. We thus consider this a "maximum accretion" case which serves as a very robust upper limit to the total accreted amount for each star. This leads to the conclusion that the concept of stellar archaeology is accurate and that the observed abundances of metal-poor stars truly reflect the chemical compositions of the time and place of their formation. Furthermore, it becomes obvious that kinematic information is vital for the identification of the lowest-metallicity stars in the Milky Way.

\section{The Nucleosynthetic Yields of the First Stars}

The metal enrichment provided by the first stars, assuming a top-heavy IMF, that may still be observable in the most metal-poor stars is schematically illustrated in Fig. 2. There are several progenitor mass ranges of SNe that would have provided different levels 
of enrichment in the early stages of the Universe. Assuming that the first SN had a mass in the pair-instability SN (PISN) range $\left(140-260 \mathrm{M}_{\odot}\right)$, the $\mathrm{Mg}$ yield is almost constant over the entire PISN mass range, which leads to a narrowly confined prediction of $[\mathrm{Mg} / \mathrm{H}]_{\min } \simeq$ -3.2 (dashed line in Fig. 1). If the first SN was in the mass range of $20-60 \mathrm{M}_{\odot}$, and of low explosion energy, low $\mathrm{Mg}$ values can be reached. The concept of a faint, mixing and fallback SN was developed by Umeda \& Nomoto (2003) to explain the two hyperFe-poor stars. Higher explosion energies are needed to explain the abundance pattern of metal-poor stars with $[\mathrm{Fe} / \mathrm{H}] \gtrsim-4.5$. We use the constraints set by observations as lower and upper limits for potential $\mathrm{Mg}$ enrichments by $\mathrm{SNe}$ in this mass range; $-5.2<[\mathrm{Mg} / \mathrm{H}]<-3.9$ (dotted lines, Fig. 1).

\section{Minimum Fe and Mg abundances in the Galaxy}

A fundamental characteristic of the Milky Way is the minimum observable metalenrichment in its stars. The existence and level of such a "metallicity floor" is governed by the Pop III IMF, thus providing us with an empirical way to constrain it. If the first stars were formed with a normal, Salpeter-like IMF, contrary to the current consensus view, there would be no minimum stellar metallicity, and truly metal-free, low-mass stars would exist. As shown in Fig. 1, interstellar accretion could not masquerade such putative primordial abundances for those stars that are observed to have sufficiently large velocities. For a top-heavy IMF, the situation is very different as described above.

Again under the assumption that the IMF was top-heavy we furthermore make use of a criterion put forward by Bromm \& Loeb (2003) for the formation of the first lowmass stars. Sufficient cooling to induce fragmentation can be reached if fine-structure line cooling through $\mathrm{C}$ and $\mathrm{O}$ is at work. $\mathrm{C}$ hereby plays the dominant role, and the minimum $\mathrm{C}$ abundance required is $[\mathrm{C} / \mathrm{H}]_{\min }=-3.5$. In combination with the maximum carbonto-iron ratio, $[\mathrm{C} / \mathrm{Fe}]_{\max }$, found in any metal-poor star (in this case, HE 1327-2326; Frebel et al. (2008)), we calculate a minimum observable metallicity in the Galaxy of $[\mathrm{Fe} / \mathrm{H}]_{\min }=-7.5$. If $[\mathrm{C} / \mathrm{H}]_{\min }$ is lower, e.g., $[\mathrm{C} / \mathrm{H}]_{\min }=-4.5$ the minimum observable metallicity would be even lower (see Fig. 1). The same was analogously done for $\mathrm{Mg}$, leading to $[\mathrm{Mg} / \mathrm{H}]_{\min }=-5.5$.

As shown in Fig. 1, the "IMF-sensitive", higher-velocity stars all have Mg abundances above the abundance floor predicted for a top-heavy Pop III IMF. We have demonstrated that accretion would not prevent the occurrence of stars with $\mathrm{Mg}$ and Fe abundances below those values. Such occurrence would refute the theoretical prediction that the first stars were very massive. However, the data is instead supporting the top-heavy theory, for the first time using the minimum observed stellar metallicity to probe the primordial IMF in a novel, near-field cosmological way. Again, we note that without proper attention to kinematics, such tests could not be carried out.

\section{Predictions for Future Metal-Poor Star Searches}

Future missions such as GAIA will enable us to extend this work by providing accurate proper motions, including most of the currently known metal-poor giants. Since our diagnostic is based on easily obtainable medium-resolution spectra, it will thus be easily applicable also to future large-scale surveys for these fossil stars.

Provided that they have sufficiently high space velocities to avoid significant accretion, we predict that future surveys should be able to identify stars with Fe abundances below the current record of $[\mathrm{Fe} / \mathrm{H}] \sim-5.5$. The recent discovery of two stars with $[\mathrm{Fe} / \mathrm{H}]<-5.0$ 


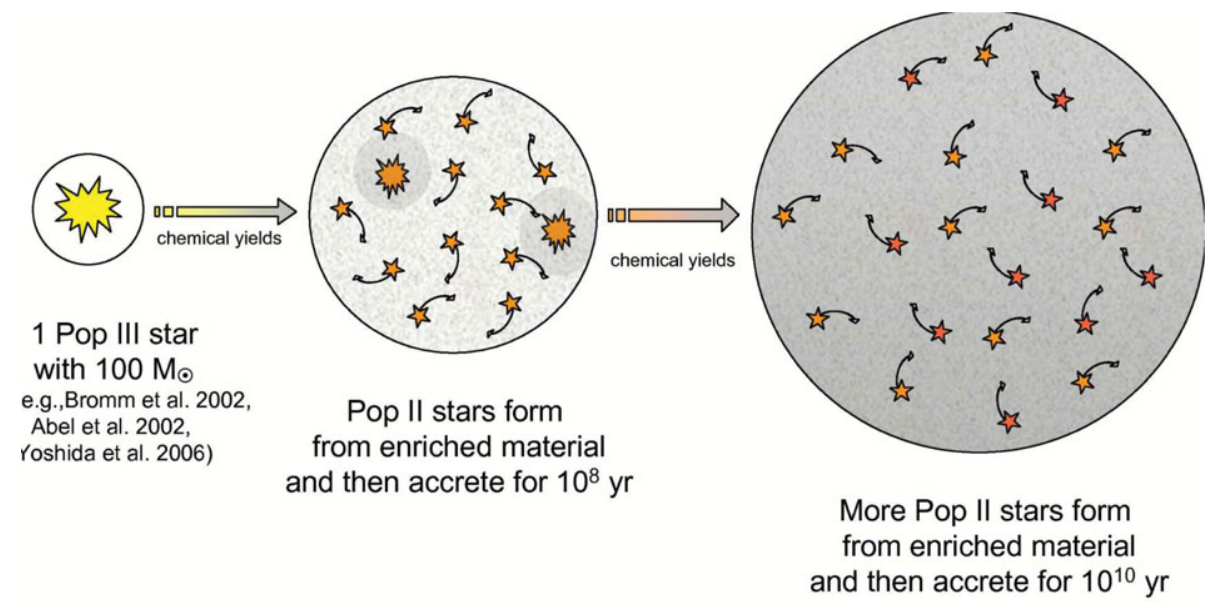

Figure 2. Schematic view of the build-up of the Milky Way under the assumption that the IMF was top-heavy. According to cosmological simulations, one massive Pop III star forms in each $10^{6} \mathrm{M}_{\odot}$ minihalo $(\mathrm{z} \sim 30-20)$ which provides the first chemical enrichment in the Universe. As the minihalos merge into a $10^{8} \mathrm{M}_{\odot}$ atomic cooling halo $(\mathrm{z} \sim 15-10)$, these metals provide a metallicity floor from which the next generation of stars form (= first Pop II stars). The more massive objects explode as $\mathrm{SNe}$, thus contributing additional metals to the interstellar medium. The first low-mass stars begin to accrete material from the sparsely enriched medium for 100 million years. As the system evolves into the $10^{12} \mathrm{M}_{\odot}$ Milky Way $(\mathrm{z} \sim 1-0)$, accretion of more metal-rich material now takes place over $10^{10}$ years.

has already demonstrated that such objects can indeed be found with current observational techniques. Based on spectrum synthesis calculations it can be estimated that suitably cool giants should have at least one detectable $\mathrm{Mg}$ and $\mathrm{Fe}$ line at abundances as low as $[\mathrm{Mg} / \mathrm{H}] \sim-6.5$ and $[\mathrm{Fe} / \mathrm{H}] \sim-8.0$, respectively. This possibility is directly relevant for the science goals of the next-generation $30 \mathrm{~m}$-class optical telescopes. Selecting suitable candidates for this task will unceasingly rely on our ability to combine chemical abundance analyses with kinematic information.

\section{References}

Bromm, V. \& Loeb, A. 2003, Nature, 425, 812

Bromm, V., Coppi, P. S., \& Larson, R. B. 2002, ApJ 564, 23

Frebel, A., Aoki, W., Christlieb, N., Ando, H., Asplund, M., Barklem, P. S., Beers, T. C., Eriksson, K., Fechner, C., Fujimoto, M. Y., Honda, S., Kajino, T., Minezaki, T., Nomoto, K., Norris, J. E., Ryan, S. G., Takada-Hidai, M., Tsangarides, S., \& Yoshii, Y. 2005, Nature, 434,871

Frebel, A., Johnson, J. L., \& Bromm, V. 2007, MNRAS, 380, L40

Iben, I. 1983, Memorie della Societa Astronomica Italiana, 54, 321

Johnston, K. V. 1998, ApJ, 495, 297

Umeda, H. \& Nomoto, K. 2003, Nature, 422, 871 